Vietnam Journal of Mechanics, VAST, Vol.34, No. 4 (2012), pp. $311-320$

\title{
MOLECULES SENSING LAYER DESIGN OF PIEZORESISTIVE CANTILEVER SENSOR FOR HIGHER SURFACE STRESS SENSITIVITY
}

\author{
Tsung-I Yin, Tien Anh Nguyen \\ University of Freiburg, Germany
}

\begin{abstract}
This paper reports on molecular sensing layer design of a piezoresistive cantilever sensor for higher surface stress sensitivity. The proposed analyses show that the previous understanding of piezoresistive cantilevers for surface stress measurement requires reconsideration for a cantilever utilizing polycrystalline silicon as a piezoresistor. The integration of the molecular sensing layer stripe pattern design to the cantilever effectively improves the piezoresistive output and utilizes the full sensing area of the cantilever surface. The proposed sensing layer design can be effectively integrated to current piezoresistive cantilever sensors to improve sensor performance in biochemical assays.

Keyword: piezoresistive cantilever sensors, surface stress sensitivity, biochemical assays.
\end{abstract}

\section{INTRODUCTION}

Surface stress is a well-known property of a solid surface [1]. Recently, a cantilever has been applied as a versatile biochemical sensor for measurement of surface stress induced by hybridization of DNA, antibody-antigen binding, small ion detection, toxic gas detection, and intermolecular interaction of self-assembling monolayers [2]. Different detection methods, including optical levers [3], as well as piezoresistive [4], piezoelectric [5], capacitance [6], and MOSFET devices [7], have been developed to measure the cantilever deflection induced by surface stress change. Due to the prevalence of piezoresistors in the current sensor market and their inherent advantages in microsystem integration, such as in microfluidic parts, piezoresistive detection is highly attractive in cantilever sensor design. However, the accompanying electrical noise in sensor operation, e.g. $1 / f$ noise, poses a serious limitation to their application in performing biochemical assays [8]. The minimum detectable surface stress for an optimal piezoresistive cantilever sensor should be in the range of $1 \mathrm{mN} / \mathrm{m}$ [9], which is at least one order of magnitude higher than that by optical lever detection. Hence, a primary driver for piezoresistive cantilever sensor design is to improve the signal-to-noise ratio for surface stress measurement.

As an intrinsic characteristic of isotropic surface stress loading, cantilever mechanics differs from the widely-studied cases in AFM-based sensors for tip force measurement [10, 11]. The effect of isotropic stress needs to be considered in sensor design. For piezoresistive cantilever sensors having p-doped single-crystal silicon as the piezoresistor (i) the 
region near the clamped edge of the cantilever is more effective for surface stress measurement $[12,13]$ due to higher piezoresistance signal in this region. Also, (ii) a wide and short cantilever shape rather than the more common long and narrow cantilever shape is more desirable for surface stress measurements [13] because of the higher $S / N$ ratio of the piezoresistance signal associated with this geometry. These conclusions demonstrate that the sensor region near the clamped edge can effectively amplify the piezoresistance signal. These examples illustrate that the reasons for the increases in piezoresistance signal are intrinsically different. For surface stress measurements, the predominant transverse piezoresistive effect, which could be reasonably neglected in an AFM-based piezoresistive sensor, will be minimized along the clamped edge to maintain the longitudinal piezoresistive effect. In addition, due to the complex mechanics caused by the clamped edge, a region away from the clamped edge is a better choice for surface stress measurement, where an unmodified Stoney's equation can be effectively applied to provide for a simple relation of surface stress loading vs. cantilever deflection [14].

One possible way to overcome the mechanical complexity caused by the clamped edge and effectively use more of the cantilever sensor is by using an n- doped singlecrystalline silicon as a piezoresistor [12]. Due to the inverse relation between the longitudinal and transverse piezoresistive coefficients for n-type doped $\mathrm{Si}$, the piezoresistance signal can be kept well below surface stress loading [12]. However, for a polysilicon piezoresistor, which is more amenable in a CMOS fabrication process, the relation between the longitudinal and transverse piezoresistive coefficients differs from that for single crystal silicon [15]. Modeling focuses on polysilicon as a piezoresistor to more effectively use the sensing area of the cantilever for higher surface stress sensitivity is necessary.

In this work, we analyze the piezoresistive effect for a polysilicon cantilever under surface stress loading. A stripe pattern design as a molecular sensing layer [16] is integrated into the piezoresistive cantilever sensor design. Fabrication of such a stripe pattern integrated cantilever sensor chip was implemented by a standard CMOS process.

\section{THEORETICAL BACKGROUND}

\subsection{Modeling of the piezoresistive effect}

To perform liquid- and gas-phase surface stress measurements, cantilever sensors are generally composed of a multilayer structure, using the bottom and top insulation layers to encapsulate the piezoresistor, with a molecular sensing layer on top of the cantilever $[9,16]$. Fig. 1 shows a multilayer cantilever with an embedded piezoresistor, considering only the mechanical behavior of the free end of the cantilever. Assuming that the applied surface stress, $\sigma_{s}$, due to the effect of gas-phase molecular adsorption is isotropic, the magnitude of the induced plane stress on the piezoresistor, i.e. the longitudinal stress, $\sigma_{l}$, and transverse stress, $\sigma_{t}$, are equal. Under the free boundary condition where $\sigma_{z}=0$ in a piezoresistor, $\sigma_{l}$ is equal to $E\left(\varepsilon_{l}+\nu \varepsilon_{t}\right) /\left(1-\nu^{2}\right)$ and $\sigma_{t}$ is $E\left(\varepsilon_{t}+\nu \varepsilon_{l}\right) /\left(1-\nu^{2}\right)$, where $E$ is the Young's modulus, $\nu$ is the Poisson's ratio, and $\varepsilon_{l}$ and $\varepsilon_{t}$ are the longitudinal strain and transverse strain, respectively. The normal strain, $\varepsilon_{z}$, in the piezoresistor is equal to $-\nu\left(\sigma_{l}+\sigma_{t}\right) / E$.

By neglecting the boundary condition of the clamped edge, the change of resistance 
in the piezoresistor can be expressed as $\Delta R / R=F_{\text {par }} \varepsilon_{l}+F_{\text {per }} \varepsilon_{t}+\varepsilon_{l}-\varepsilon_{t}-\varepsilon_{z}$, where $F_{\text {par }}=E\left(\pi_{l}+\nu \pi_{t}\right) /\left(1-\nu^{2}\right)$ and $F_{p e r}=E\left(\pi_{t}+\nu \pi_{l}\right) /\left(1-\nu^{2}\right)$ are gauge factors in the longitudinal and transverse directions [17]. They are related to the longitudinal and transverse piezoresistive coefficients $\pi_{l}$, and $\pi_{t}$, respectively. Furthermore, $\Delta R / R$ can be further simplified as:

$$
\frac{\Delta R}{R}=\left(F_{p a r}+\frac{1}{(1-\nu)}\right) \varepsilon_{l}+\left(F_{p e r}+\frac{(1-2 \nu)}{(1-\nu)}\right) \varepsilon_{t}
$$

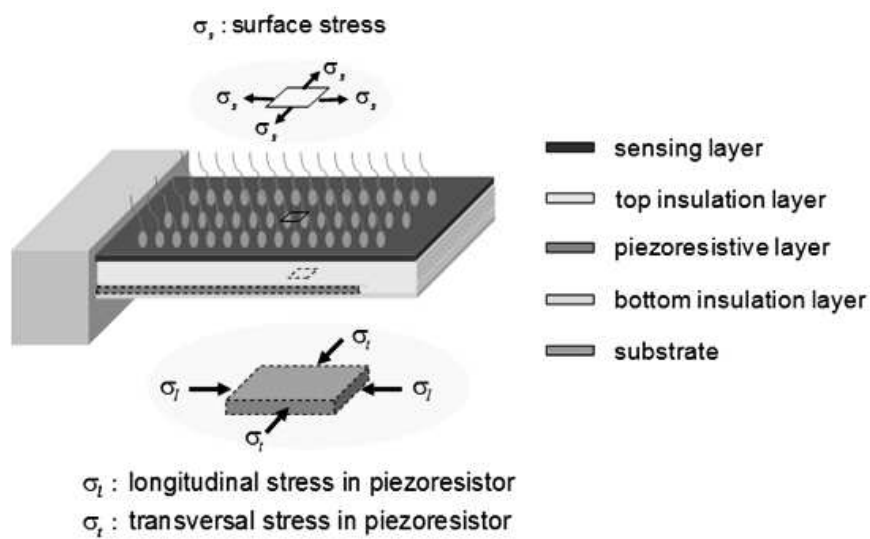

Fig. 1. Schematic diagram of the induced plane stress in the embedded piezoresistor for a multilayer cantilever under surface stress loading from molecular adsorption.

The surface stress sensitivity is defined by $\Delta R / R / \sigma_{s}$. Therefore, increased $|\Delta R / R|$ is desirable in cantilever sensor design. According to the analytical model and measured data [15], the longitudinal gauge factor for p-doped polysilicon is positive for doping concentration levels common for piezoresistors, whereas the transverse gauge factor is negative. Conversely, for n-doped polysilicon the longitudinal gauge factor is negative, whereas the transverse gauge factor is positive. The peak gauge factor, $F_{p a r}$, of p-doped polysilicon is about +38 , whereas for $F_{\text {per }}$ it is about -17 . For n-type polysilicon, the peak gauge factor, $F_{p a r}$, is about -24 , whereas for $F_{p e r}$ it is about 15 . Compared to a piezoresistor of singlecrystalline silicon, the piezoresistive effect of polysilicon is reversed in the case of n-type doping [12]. This reversal may originate from grain boundaries and the grain size effects of polysilicon [15]. Therefore, estimation of $\pi_{l}$ and $\pi_{t}$ in p-type or n-type doped silicon for facilitating surface stress measurements is inappropriate in this case $[12,16]$. Based on Eq. (1), as $\varepsilon_{l} \approx \varepsilon_{t}$, piezoresistance is dramatically attenuated due to the combined piezoresistive effects in the longitudinal and transverse directions. This effect is the same as for the cantilever sensor using p-type silicon as the piezoresistor $[12,16]$. However, this drawback could become advantageous if the strain relation is $\varepsilon_{l} \approx-\varepsilon_{t}$. 


\subsection{Molecular sensing layer design}

Fig. 2 (a) shows the cross sectional view of the multilayer cantilever in Fig. 1. The mechanical strain in the piezoresistor, $\varepsilon_{l}$ and $\varepsilon_{t}$, can be further expressed by a proposed mechanical model [16].

$$
\left[\begin{array}{l}
\varepsilon_{l}(\mathrm{z}) \\
\varepsilon_{t}(\mathrm{z})
\end{array}\right]=\frac{1}{\operatorname{det}\left(-A D+B^{2}\right)}\left[-D N_{s}+B M_{s}+\mathrm{z}\left(-B N_{s}+A M_{s}\right)\right]
$$

where $A$ is the extensional stiffness, $A=\sum_{j=1}^{4} Q_{j}\left(h_{j}-h_{j-1}\right), h_{j}$ is the position of the layer surface respect to the mid plane of cantilever, and $Q_{j}$ is the elastic constants determined by the Young's modulus $E_{j}$ and Poisson's ratio $\nu_{j}$ of structure layer $j$, and $Q_{j}=E_{j} / 1-\nu_{j}^{2}\left[\begin{array}{lll}1 & \nu_{j} ; \nu_{j} & 1\end{array}\right] . B$ is the coupling stiffness, $B=1 / 2 \sum_{j=1}^{4} Q_{j}\left(h_{j}^{2}-h_{j-1}^{2}\right)$, and $D$ is the bending stiffness of the cantilever, $D=1 / 3 \sum_{j=1}^{4} Q_{j}\left(h_{j}^{3}-h_{j-1}^{3}\right)$. The resultant force, $N_{s}=\left[\sigma_{s} ; \sigma_{s}\right]$, and $M_{s}$ is the resultant moment from surface stress loading, $M_{s}=h_{4}\left[\sigma_{s} ; \sigma_{s}\right]$.

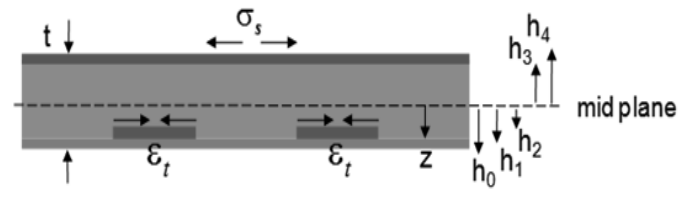

(a)

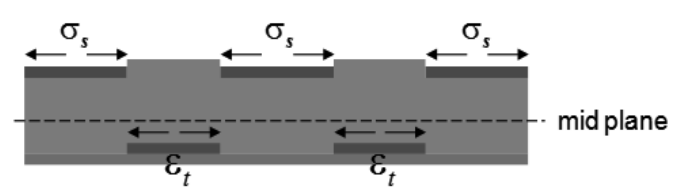

(b)

Fig. 2. Cross sectional view of the multilayer cantilever with (a) normal pattern and (b) stripe pattern sensing layer on cantilever surface.

Eq. (2) indicates that the magnitude of $\varepsilon_{l}$ and $\varepsilon_{t}$ depends only on the total thickness, $t$, and the piezoresistor position, $z$, relative to the mid plane of the cantilever. Smaller $\mathrm{t}$ and larger $z$ lead to higher piezoresistor strain, i.e., higher surface stress sensitivity $\Delta R / R / \sigma^{s}$. However, the attenuation of piezoresistance signal described in Eq. (1) cannot be improved merely by the optimal design of $t$ and $z$. In contrast to the traditional sensing layer design, Fig. 2 (b) shows a stripe pattern sensing layer formed on the cantilever surface. Due to the gap between the different sensing layers, the direction of $\varepsilon_{t}$ is reversed under the same surface stress loading, as the compressive stress between the sensing layer striped regions induces tensile stress in the piezoresistant layer. Fig. 3 (a) and (b) show the finite element analysis (ANSYS 11.0) for $\varepsilon_{l}$ and $\varepsilon_{t}$ along the x-axis in the piezoresistor with normal and stripe sensing layer patterns for a cantilever of length $50 \mu \mathrm{m}$, width $30 \mu \mathrm{m}$, and thickness $1.3 \mu \mathrm{m}$. The applied surface stress loading $\sigma_{s}$ is $1 \mathrm{~N} / \mathrm{m}$ on the cantilever surface with the piezoresistor on the bottom side of the cantilever. For the normal pattern, the surface stress loading is distributed uniformly on the entire area of the cantilever. The effect of the clamped edge can be observed in the value of $\varepsilon_{l}$ and $\varepsilon_{t}$, where $\varepsilon_{l} \approx \varepsilon_{t}$ in the region farthest away from the clamped edge, indicating that the longitudinal and transverse stresses converge, as the distance from the cantilever increases, reducing sensitivity and the effective area of the cantilever. For the striped pattern with a $5 \mu \mathrm{m}$ gap, the surface 


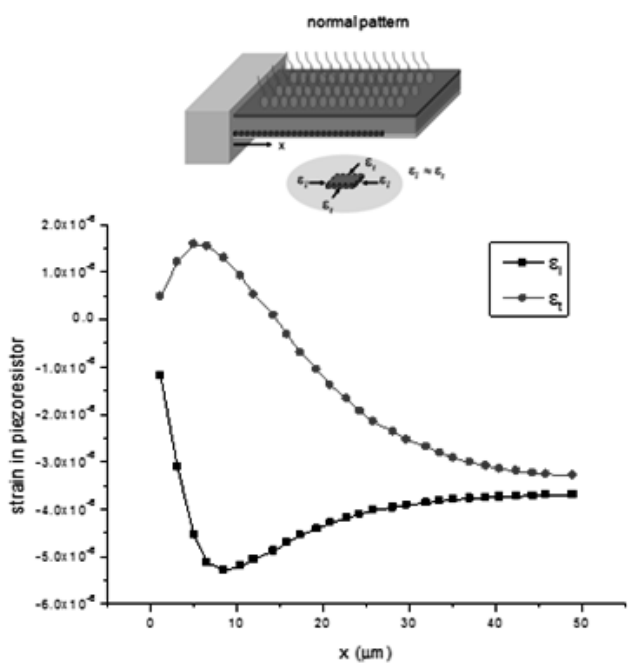

(a)

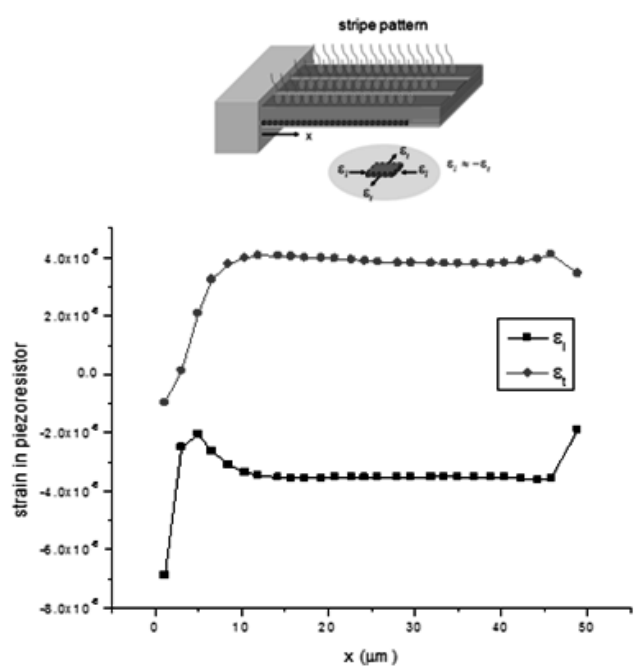

(b)

Fig. 3. Finite element analysis simulation of the mechanical strain in a piezoresistor with (a) normal pattern and (b) stripe pattern on a $50 \mu \mathrm{m}$ long, $30 \mu \mathrm{m}$ wide, and $1.3 \mu \mathrm{m}$ thick cantilever. The applied surface stress loading is $1 \mathrm{~N} / \mathrm{m}$. The material properties were from [16].

stress loading is applied to alternating regions areas of the cantilever surface. Finite element analysis indicates that the relation between $\varepsilon_{l}$ and $\varepsilon_{t}$ is effectively reversed to $\varepsilon_{l} \approx-\varepsilon_{t}$, increasing the sensitivity and the usable area of the cantilever. Thus, the piezoresistance performance, $\Delta R / R$, can be significantly improved by the stripe pattern design.

\section{EXPERIMENTAL SECTION}

\subsection{Fabrication of a CMOS cantilever sensor}

A CMOS cantilever sensor was fabricated based on the proposed design concept. Fig. 4 (a-c) illustrates the fabrication process by TSMC, using a $0.35 \mu \mathrm{m} 2 \mathrm{P} 4 \mathrm{M}(2 \mathrm{poly} / 4$ metal) CMOS process [18]. Two polysilicon layers and four metal layers in the CMOS process facilitate a versatile cantilever sensor structural design. A multilayer structure was deposited, where $\mathrm{n}^{+}$doped polysilicon (poly 2) at a concentration of $1 \sim 5 \times 10^{20} \mathrm{at} / \mathrm{cm}^{3}$ was used for the piezoresistive layer. Metal layers 1-4 and TEOS inter-metal dielectric layer were stacked as structural layers and as a metal line connection. The trench was formed by anisotropic etching of TEOS using a metal layer as a hard mask. In post-CMOS micromachining, a $\mathrm{Ti} / \mathrm{Au}$ layer as the molecular sensing layer on the cantilever was deposited and protected using a thicker coating of photoresist. Isotropic dry etching of the silicon substrate was used to release the cantilever structure. Fig. 5 (a) shows the SEM photo of the cantilever structure. A single cantilever is $50 \mu \mathrm{m}$ in length, $30 \mu \mathrm{m}$ in width, and $2.7 \mu \mathrm{m}$ in thickness. The SEM image shows that the residual film stress in the cantilever is well compensated to maintain the flatness of the structure. The dimensions of the embedded 

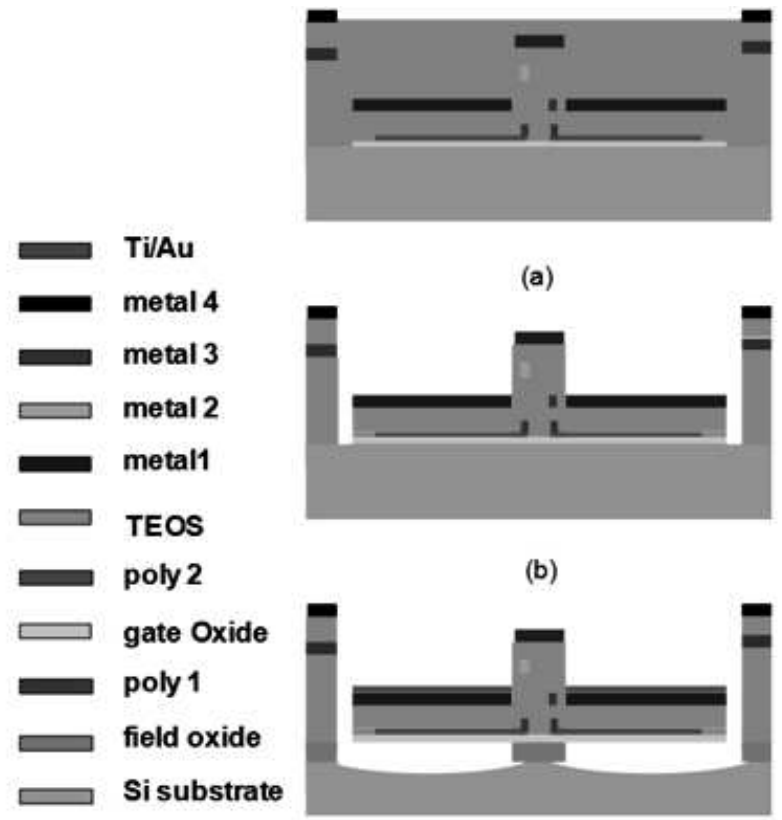

(b)

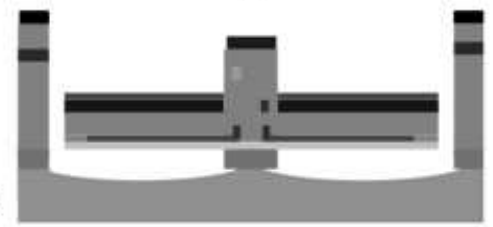

(c)

Fig. 4. CMOS fabrication process of the cantilever sensor, including (a) structure layer stacking, (b) trench etching, and (c) molecular sensing layer deposition and cantilever structure release after post-CMOS micromachining.

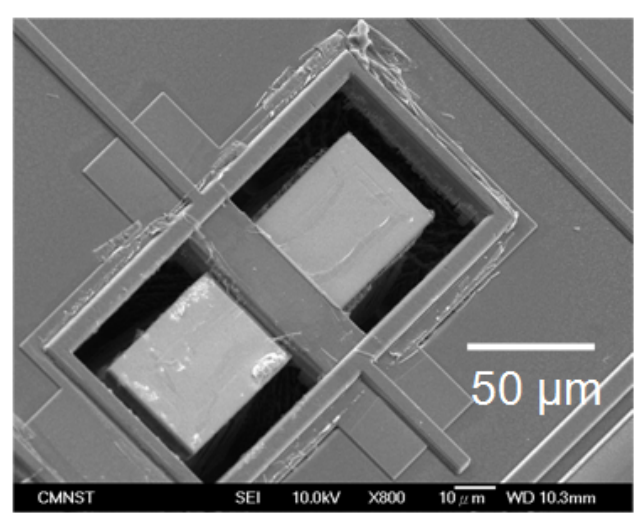

(a)
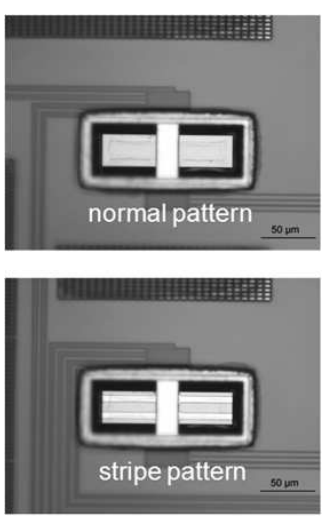

(b)

Fig. 5. (a) SEM image of the released cantilever structure. The cantilever length is $50 \mu \mathrm{m}$, width, is $30 \mu \mathrm{m}$, and thickness is $2.7 \mu \mathrm{m}$. (b) The normal and stripe pattern sensing layer on the CMOS cantilever surface. 
piezoresistor within the cantilever are $40 \mu \mathrm{m}$ in length and $3 \mu \mathrm{m}$ in width. The measured resistance of the single piezoresistor is approximately $1.84 \mathrm{k} \Omega$. Fig. 5 (b) shows the normal and stripe design patterns of the gold layer on the cantilever as a molecular sensing layer.

\subsection{Bridge circuit design}

Fig. 6 shows the arrangement of the cantilever sensor as a Wheatstone bridge for surface stress measurement. For liquid-phase measurement in this work, a microfluidic channel cover made of polydimethylsiloxane (PDMS) was integrated within the CMOS cantilever chip using a stamp-and-stack bonding technique [19]. Fully symmetric can-

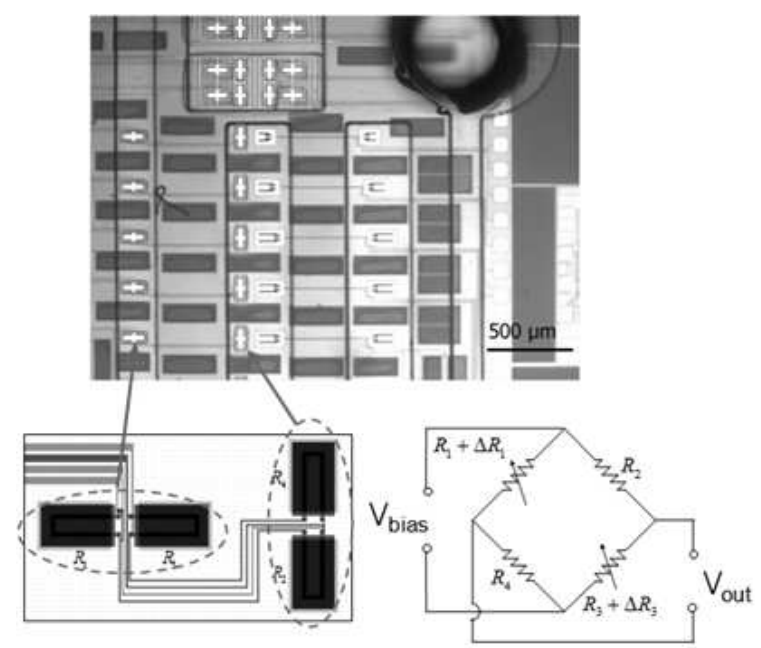

Fig. 6. The arrangement of the cantilever sensor as a Wheatstone bridge for surface stress measurement. Fully symmetric cantilevers adopted as the reference cantilevers $\left(R_{2}\right.$ and $\left.R_{4}\right)$ and sensing cantilevers $\left(R_{1}\right.$ and $\left.R_{3}\right)$ were placed in an isolated microfluidic channel.

tilevers were adopted as the sensing cantilevers $\left(R_{1}\right.$ and $\left.R_{3}\right)$, and reference cantilevers $\left(R_{2}\right.$ and $R_{4}$ ) were placed within the isolated microfluidic channel. By injecting the analyte into the channel having the sensing cantilever, a change in resistance, $\Delta R_{1}$ and $\Delta R_{3}$ was induced by the adsorption of analyte on the cantilever surface. Using this bridge circuit design, $V_{\text {out }} / V_{\text {bias }}=1 / 2 \Delta R / R$, where $V_{\text {bias }}$ is the bias voltage of the bridge circuit.

\subsection{Sensor operation condition}

Due to electrical and thermo-mechanical noise induced by the embedded piezoresistor during sensor operation, the bias voltage $V_{\text {bias }}$ must be chosen to improve the signalto-noise ratio. The dominant noise of the cantilever sensor when operated at low frequency is $1 / f$ noise, and is proportional to $V_{\text {bias }}^{2}$ [8]. The power consumption of the bridge circuit, which is directly related to the induced temperature in the cantilever, is also proportional to $V_{\text {bias }}^{2}$ [16]. Therefore, low $V_{\text {bias }}$ is desirable in senor operation to reduce noise. To prevent additional thermal noise in the bridge circuit, the induced temperature in the cantilever for either normal or stripe pattern sensing layers should be identical. Fig. 7 shows that the 


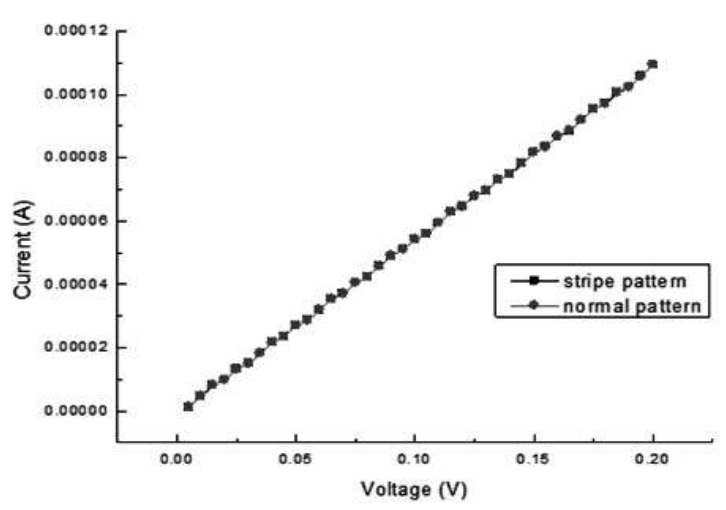

Fig. 7. The I-V curve of the bridge circuit for cantilevers having normal or stripe pattern sensing design. layers.

I-V curve of the bridge circuit with normal or stripe pattern has equivalent resistance, or the same thermo-mechanical bridge circuit characteristics during low voltage operation. Thus, $0.1 \mathrm{~V}$ is selected as $V_{\text {bias }}$ in the following measurement.

\section{RESULTS AND DISCUSSIONS}

According to Eqs. (1) and (2), the stripe pattern design is not limited to a specific layer thickness or piezoresistor position as long as the adsorption layer is not on top of the piezoresistor as shown in Fig. 2 (b). A cantilever design with smaller $t$ and larger $z$ has higher piezoresistor strain, thus, higher surface stress sensitivity. Due to the linear relationship between the magnitude of the surface stress loading, $\sigma_{s}$, and piezostrain, $\varepsilon_{l}$ and $\varepsilon_{t}$, in the area away from the clamped edge of cantilever, Stoney's equation can be effectively applied to the proposed design to estimate the relation between surface stress loading and the sensor signal.

Assuming tensile surface stress loading on the cantilever as shown in Fig. 1, Tab. 1 polysilicon films as the piezoresistor at different positions relative to the midplane. The results indicate that the cantilever sensor $|\Delta R / R|$ for a stripe pattern design can be improved by prudent placement of the piezoresistor above or below the mid plane. For this design, the main cantilever surface becomes an effective sensing area for surface stress measurement. Depending on the polysilicon doping concentration, the gauge factor could be further optimized for higher piezoresistive output [15]. summarizes the relation of $\Delta R / R$ with different

The proposed sensing layer design can be integrated with other techniques to increase surface stress loading for improved cantilever sensor performance. Gold grain size and surface discontinuities affect the final structure of the $\mathrm{Ti} / \mathrm{Au}$ self-assembly layer and thus have a strong influence on the magnitude of the induced surface stress [1]. The morphology, adhesion, and cleanliness of the gold sensing surface significantly influence the performance of the cantilever sensor [1]. Other surface immobilization techniques, e.g. 
Table 1. The relation of $\Delta R / R$ with different polysilicon films as the piezoresistor at different positions relative to the midplane

\begin{tabular}{|c|c|c|c|c|c|}
\hline & $F_{\text {par }}$ & $F_{\text {per }}$ & $\varepsilon_{l}$ & $\varepsilon_{t}$ & $\Delta R / R$ \\
\hline case 1 & + & - & + & - & + \\
\hline case 2 & - & + & + & - & - \\
\hline case 3 & + & - & - & + & - \\
\hline case 4 & - & + & - & + & + \\
\hline
\end{tabular}

layer-by-layer assembly and coadsorption of long-chain thiols along with the $\mathrm{Ti} / \mathrm{Au}$ to increase the surface density, could also enhance the magnitude of surface stress loading [1]. Such techniques for enhancing the surface stress loading on the cantilever surface are expected to further improve piezoresistor performance in a biochemical assay.

Case 1 is for a p-type doping piezoresistor above the mid plane $(z>0)$, case 2 for an n-type doping piezoresistor above the mid plane $(z>0)$, case 3 is for a p-type doping piezoresistor below the mid plane $(z<0)$, and case 4 is for an n-type doping piezoresistor below the mid plane $(z<0) . F_{p a r}$ and $F_{p e r}$ are the gauge factors of the piezoresistor in the longitudinal and transverse directions, respectively. $\varepsilon_{l}$ and $\varepsilon_{t}$ are the longitudinal and transverse strain in the piezoresistor, respectively.

\section{CONCLUSIONS}

This work proposes a molecular sensing layer design for a piezoresistive cantilever sensor having higher surface stress sensitivity. The finite element modeling and analysis indicate that the piezoresistive effect in doped polysilicon is different from that in single-crystalline silicon under the same surface stress loading. The attenuation of piezoresistance signal induced by the isotropic surface stress loading on a cantilever sensor can be effectively overcome by altering the sensing layer design. By stripe-patterning the sensing layer, the relation between longitudinal and transverse piezoresistor strain can be reversed to improve the piezoresistive output of the cantilever sensor and increases the effective sensing area of the cantilever surface. The proposed design is not limited by the type of doped polysilicon or the position of the piezoresistor in the cantilever, permitting more versatile piezoresistive cantilever sensor designs. Stripe-patterning can also be effectively integrated into current surface modification techniques to improve surface stress loading for improved cantilever sensor performance. Other mechanical strain-based detection methods, e.g. MOSFET strain sensors [7], could also adopt this design for higher surface stress sensitivity.

\section{ACKNOWLEDGEMENT}

The authors would like to thank Chun Chang and Victor Vartanian for providing valuable input in preparing this manuscript. 
Tsung-I Yin, Tien Anh Nguyen

\section{REFERENCES}

[1] A. W. Adamson, Physical Chemistry of Surface (Wiley, New York, 1990).

[2] Hai-Feng Ji and Benjamin D. Armon, Approaches to Increasing Surface Stress for Improving Signal-to-Noise Ratio of Microcantilever Sensors, Analytical Chemistry, 82 (5), (2010), 16341642.

[3] J. Fritz, M. K. Baller, H. P. Lang, H. Rothuizen, P. Vettiger, E. Meyer, H. -J. Güntherodt, Ch. Gerber,and J. K. Gimzewski, Translating Biomolecular Recognition into Nanomechanics, Science, 288 (5464), (2000), 316-318.

[4] A. Boisen, J. Thaysen, H. Jensenius, and O. Hansen, Environmental sensors based on micromachined cantilevers with integrated read-out, Ultramicroscopy, 82, (2000), 11-16.

[5] H. Yamada, H. Itoh, S. Watanabe, K. Kobayashi, and K. Matsushige, Scanning near-field optical microscopy using piezoelectric cantilevers, Surface and Interface Analysis, 27, (1999), 503-506.

[6] T. Thundat, P. I. Oden, and R. J. Warmack, Microcantilever sensors, J. Microscale Thermophys. Eng, 1, (1997), 185-199.

[7] G. Shekhawat, S.-H. Tark, and V. P. Dravid, MOSFET-Embedded Microcantilevers for Measuring Deflection in Biomolecular Sensors, Science, 311, (2006), 1592-1595.

[8] J. R. Mallon, Jr., A. J. Rastegar, A. A. Barlian, M. T. Meyer, T. H. Fung, and B. L. Pruitt, Low $1 / \mathrm{f}$ noise, full bridge, microcantilever with longitudinal and transverse piezoresistors, Apply Physics Letter, 92, (2008), 033508.

[9] P. A. Rasmussen, J. Thaysen, O. Hansen, S. C. Eriksen, and A. Boisen, Optimised cantilever biosensor with piezoresistive read-out, Ultramicroscopy, 97, (2003), 371-376.

[10] M. Tortonese, R. C. Barrett, and C. F. Quate, Atomic resolution with an atomic force microscope using piezoresistive detection, Apply Physics Letter, 62, (1993), 834-836.

[11] J. A. Harley and T. W. Kenny, 1/f noise considerations for the design and process optimization of piezoresistive cantilevers, J. Microelectromechanical Systems, 9, (2000), 226-235.

[12] P. A. Rasmussen, O. Hansen, and A. Boisen, Cantilever surface stress sensors with singlecrystalline silicon piezoresistors, Apply Physics Letter, 8, (2005), 203502.

[13] F. T. Goericke and W. P. King, Modeling Piezoresistive Microcantilever Sensor Response to Surface Stress for Biochemical Sensors, IEEE Sensors Journal, 8, (2008), 1404-141.

[14] J. E. Sader, Surface stress induced deflections of cantilever plates with applications to the atomic force microscope: Rectangular plates, Journal of Apply Physics, 89, (2001), 2911-2921.

[15] P. J. French and A. G. R. Evans, Piezoresistance in polysilicon and its applications to strain gauges, Solid-State Electronics, 32, (1989), 1-10.

[16] S. M. Yang and T. I. Yin, Design and analysis of piezoresistive microcantilever for surface stress measurement in biochemical sensor, Sens. Actuators B, 120, (2007), 736-744.

[17] L. Lin and W. Yun, Design optimization and fabrication of surface micromachined pressure sensors, Mechatronics, 8, (1998), 505-519.

[18] H. H. Tsai, C. F. Lin, Y. Z. Juang, I. L. Wang, Y. C. Lin, R. L. Wang, and H. Y. Lin, Multiple type biosensors fabricated using the CMOS BioMEMS platform, Sens. Actuators B, 144, (2010), 407-412.

[19] S. Satyanarayana, R. N. Karnik, and A. Majumdar, Spatially controlled microfluidics using low-voltage electrokinetics, Journal of Microelectromechanic System, 14, (2005), 392-399.

Received July 31, 2012 